\title{
Selective Excitation of Circular Helical Modes in Power-Law Index Fibers
}

\author{
S. Al-Sowayan ${ }^{1}$ \\ ${ }^{1}$ EE Department, Al Imam Mohammad Ibn Saud Islamic University, Riyadh, Saudi Arabia \\ Correspondence: S. Al-Sowayan, EE Department, Al Imam Mohammad Ibn Saud Islamic University, P. O. Box \\ 93718, Riyadh 11683, Saudi Arabia. E-mail: sssowayan@imamu.edu.sa
}

Received: December 28, 2013

Accepted: January 12, $2014 \quad$ Online Published: January 19, 2014

doi:10.5539/mas.v8n1p134

URL: http://dx.doi.org/10.5539/mas.v8n1p134

\begin{abstract}
Selective excitation of circular helical modes in power-law index fibers is shown to have an impact on their capacity. This is analyzed using a model for propagation delay variation with launch offset and angle that resulted from misalignment of source and fiber axis. Results show promising technique to improve power law-index fibers capacities.
\end{abstract}

Keywords: fiber measurements, fiber optics, powe-law index profile, propagation modes

\section{Introduction}

It is a fact that when the bandwidth demand increased by the high bandwidth applications required from communication networks, the capacity of the already installed thousands of kilometers of multimode fibers (MMF) will be a bottleneck by Raddatz (1998, 1999), Coleman (2006), Gu (2006) and McInally (2002). Despite availability of alternatives such as single mode fibers and ribbon link fibers analyzed by Hahn (1995), the cost of replacing already installed infrastructure will be high shown by Amphawan (2010). Legacy MMF with $62.5 \mu \mathrm{m}$ core can have bandwidth as low as $160 \mathrm{MHz} . \mathrm{km}$ at $850 \mathrm{~nm}$ wavelength, and the observed bandwidth can be sensitive to launch angle and offset shown by Webster (1999). If the launch condition makes perfect alignment between source and fiber axis, what is so called meridional modes will only be excited. Meridional modes are the propagation modes that follow a path cross the fiber axis. The axial mode, which is the lowest order mode, is the mode of propagation that travels along the longitudinal axis of the fiber. If there is misalignment in both angle and offset between the source and the fiber axis what is called helical modes of propagation will be excited. Helical modes are the type of modes that propagate in a helix shape inside the core of the fiber along the longitudinal direction that do not cross the fiber axis and bounded by the total internal reflection inside the core of the fiber by Murshid (2008).

In particular, low angle, on-axis power excites a selection of low-order modes which can result in excessive and unstable pulse broadening relative to over filled launch conditions by Sargent (1998). Since the bandwidth is sensitive to launch parameters, it is important to consider which groups of modes are being excited by laser sources. In this paper a computer model for propagation delay in graded index fibers was used to quantify the impact of selective excitation of circular helical modes only on link performance.

\section{Computer Model}

The model refers the coupling angle and offset from the center of the fiber to the order of helical path that the optical energy will follow, the equation of the circular helix is stated in Equation (1).

$$
\left\{\begin{array}{l}
x=A \cdot \cos t \\
y=A \cdot \sin t \\
z=b t
\end{array}\right.
$$

Where $\mathrm{x}$ and $\mathrm{y}$ are the transverse plane coardinates and $\mathrm{z}$ is the longitudinal direction of the fiber, $\mathrm{A}$ and $2 \pi \mathrm{b}$ are the radius and pitch of the helical mode that will be determined by the coupling (entrance) angle with the fiber core face, and the offset from the center of the fiber core. In graded index (GI) fibers power law profile, the common profile is the parabola shape of refractive index with the peak at the center of the fiber. Though the model general idea is valid for all power law profiles, the parabolic profile is considered here. It has been 
reported in literature such as Raddatz (Sep 1999) that during the manufacturing process of GI fibers there, a common defect that introduces a peak or dip in the value of refractive index at the center of the fiber is generated, which in turn reduces the capacity of the GI fibers when used with lasers at center launch in an underfilled launch regime. To avoid this defect, in this paper the entering rays will be offseted from the center by $10 \mu \mathrm{m}$. Without considering the defect at the center of the fiber the power-law index profile is described in Equation (2).

$$
n(r)= \begin{cases}\sqrt{n_{1}^{2}\left(1-2 \Delta\left(\frac{r}{a}\right)^{2}\right)} & \text { for } 0 \leq r \leq a \\ n_{1} \sqrt{1-2 \Delta} & \text { for } r \geq a\end{cases}
$$

Where $n_{1}$ is the refractive index of center of the core typical value for the glass is $1.5, \Delta$ is the change in the refractive index profile typically $0.01, \mathrm{r}$ is the radial position, and a is the radius of the core which is $31.25 \mu \mathrm{m}$. The coupling between the optical ray and fiber shall make an angle $(\theta)$ with the transverse plane of the fiber, to ensure no meridional modes nor elliptical helical modes are excited this angle shall make an entrance angle greater than the acceptance angle of the fiber (in this case it is 12 degrees) another condition is that the projection of the ray into the face of the fiber should be perpendicular to the radial vector from the center of the fiber to point of ray entrance to the core as shown in Figure 1. This means $\theta$ shall vary from 10 to 78 degrees, the 10 degrees are chosen for the helix to be realistic enough to propagate in the longitudinal direction. Table 1 shows for each entrance angle, measured from the transverse plane of the face of the fiber to the ray, the corresponding helical mode order parameters for a certain offset of $10 \mu \mathrm{m}$. Figure 2 gives a bigger picture of the relationship between the launch offset from the center of the fiber, the angle of entrance and the helical mode path length (normalized to the longest one) which can be calculated from the resulting helix parameters. Figure 2 clearly shows that as the offset gets larger the path of the optical pulse increases and as the angle $\theta$ becomes larger the optical pulse path decreases.

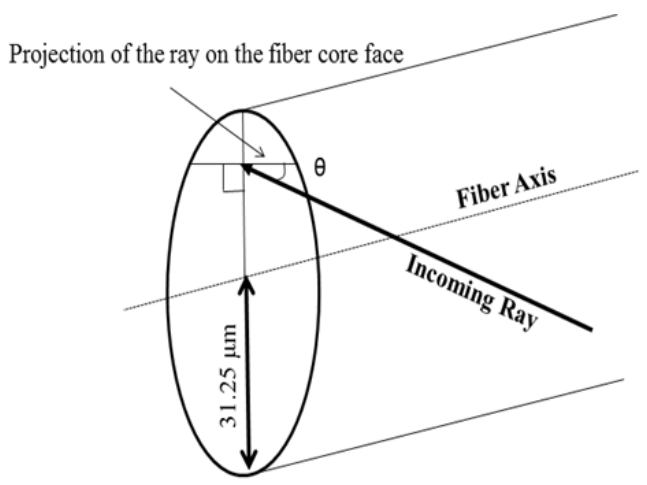

Figure 1. Misaligned coupling in both offset and angle that yield to excitation of circular helical modes

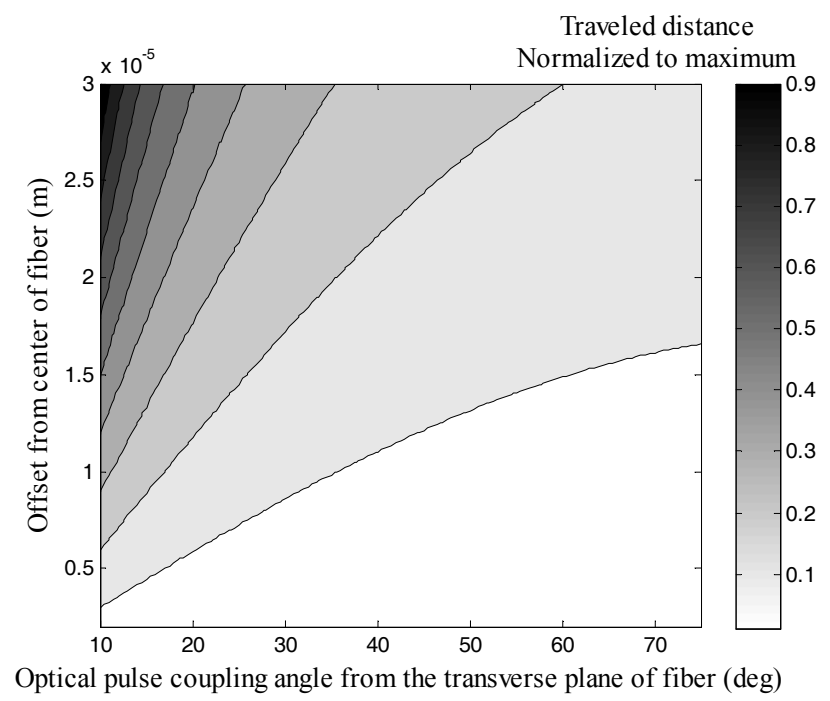

Figure 2. Distance travelled in one rotation of circular helix for a particular coupling angle and offset 
Table 1. Coupling angle with corresponding helix parameters

\begin{tabular}{cc}
\hline$\theta$ & $\begin{array}{c}\text { Normalized helix } \\
\text { parameters (A,b) }\end{array}$ \\
\hline 10 & $(10,4.39)$ \\
30 & $(10,5.41)$ \\
50 & $(10,8.05)$ \\
75 & $(10,21.8)$ \\
\hline
\end{tabular}

It is of interest to consider the experimentally generated short optical pulse by gain switching an $850 \mathrm{~nm}$ laser to produce a $\sim 200$ ps pulse shown in Figure 3. as an input parameter to the model, the model then shall consider the arrival time of the mentioned pulse by calculating the required propagation time down the fiber normalized to the maximum time. The propagation time will be calculated based on the distance the pulse shall travel and the optical pulse group velocity which will defiantly be affected by the refractive index faced by the propagating mode. Usually, in some cases, the beam waist of the optical ray entering the fiber is around $4 \mu \mathrm{m}$ by Tsekrekos (2004). Using the same beam waist and considering the optical pulse that enters the fiber with an offset from 10 $\mu \mathrm{m}$ to $14 \mu \mathrm{m}, 15-19 \mu \mathrm{m}, 20-24 \mu \mathrm{m}$, and $25-29 \mu \mathrm{m}$ offsets to avoid the defective area around the center of the fiber core. For each offset range the optical pulse is considered as a group of rays having the same $\theta$ angle (described before) with an increment of $0.1 \mu \mathrm{m}$. The distance traveled by each helical mode excited is calculated then the propagation time needed by each helical mode is calculated by dividing the distance traveled by the speed of the specific helical mode, which is the speed of light divided by the corresponding refractive index at the specified offset from Equation (1). This process is repeated for different $\theta$ angles of 10, 20,30, 40, and 50 degrees. Note that after calculating the traveled distances by each helical mode for different offsets and angles, the distances have been normalized to their maximum value.

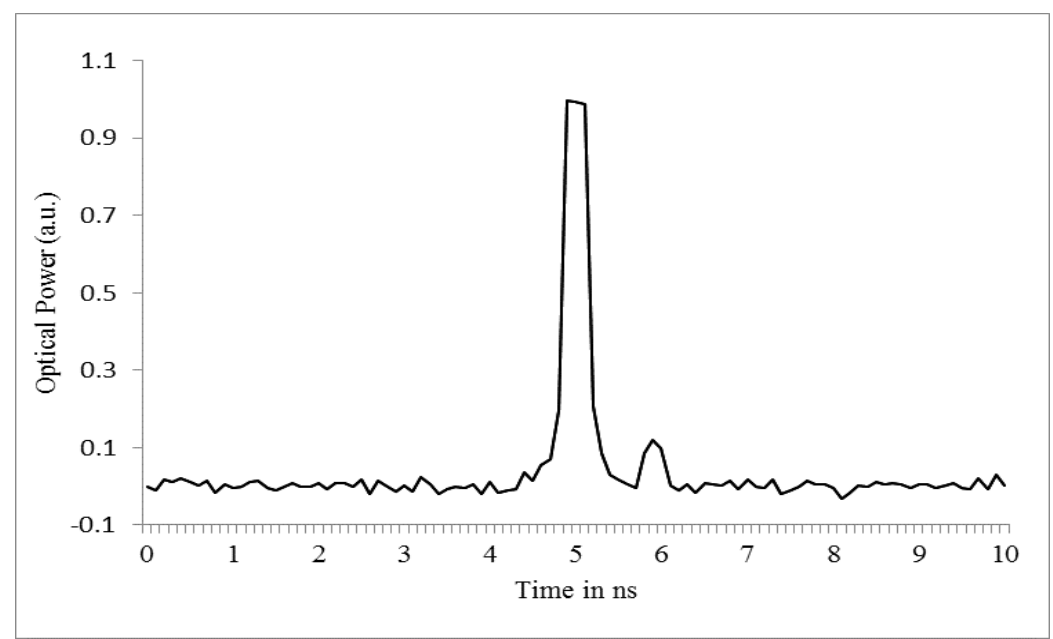

Figure 3. Optical short pulse from an 850nm laser source

For a specific angle $\theta$, the optical pulse shown in Figure 3. is then copied by each helical mode path and arrives at a reference distance at different arrival time. By adding these copies of arrived pulses at their corresponding arrival time, the result gives good approximation of the impulse response of the fiber when exciting only circular helical modes at the certain angle and offset with the mentioned beam waist.

\section{Results and Discussion}

Figure 4 shows the result of the response of the $62.5 / 125 \mu \mathrm{m}$ GI fiber when exciting only circular helical modes at $\theta=10,30$, and 50 degrees tilt from the transverse plane of the fiber core perpendicular to the radial vector with offsets of 10-14 $\mu \mathrm{m}$ in Figure 4 (a), and 25-29 $\mu \mathrm{m}$ in Figure 4(b). It is clear from Figure 4 that as $\theta$ angle gets smaller the response gets narrower, and as the offset gets larger the response gets also narrower. 
(a)

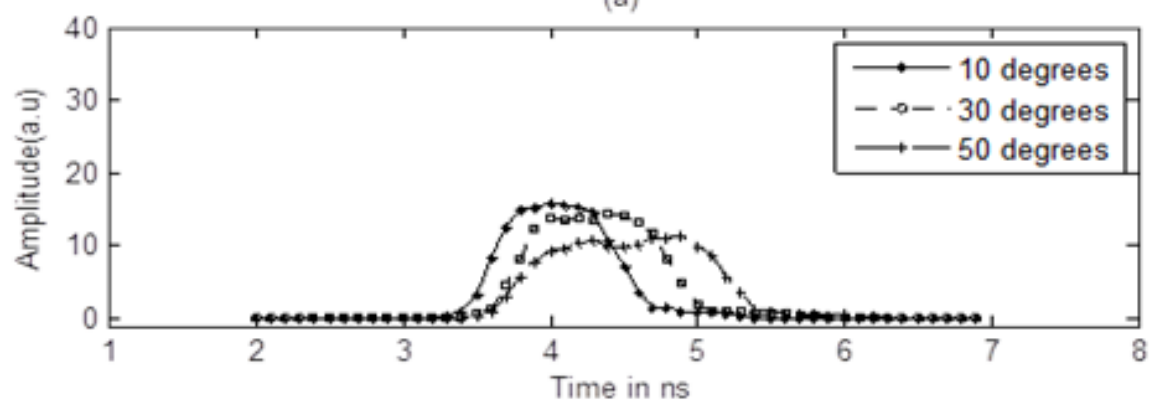

(b)

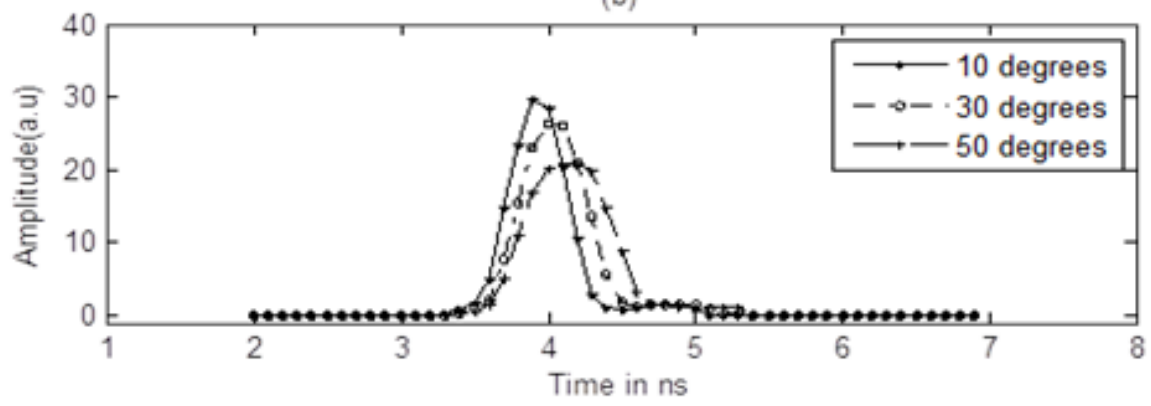

Figure 4. Optical $850 \mathrm{~nm}$ pulse response with $4 \mu \mathrm{m}$ beam waist of a $62.5 / 125 \mu \mathrm{m}$ fiber with a tilt $\theta$ angle of 10 , 30, 50 degrees at an offset of (a) 10-14 $\mu \mathrm{m}$ and (b) $25-29 \mu \mathrm{m}$

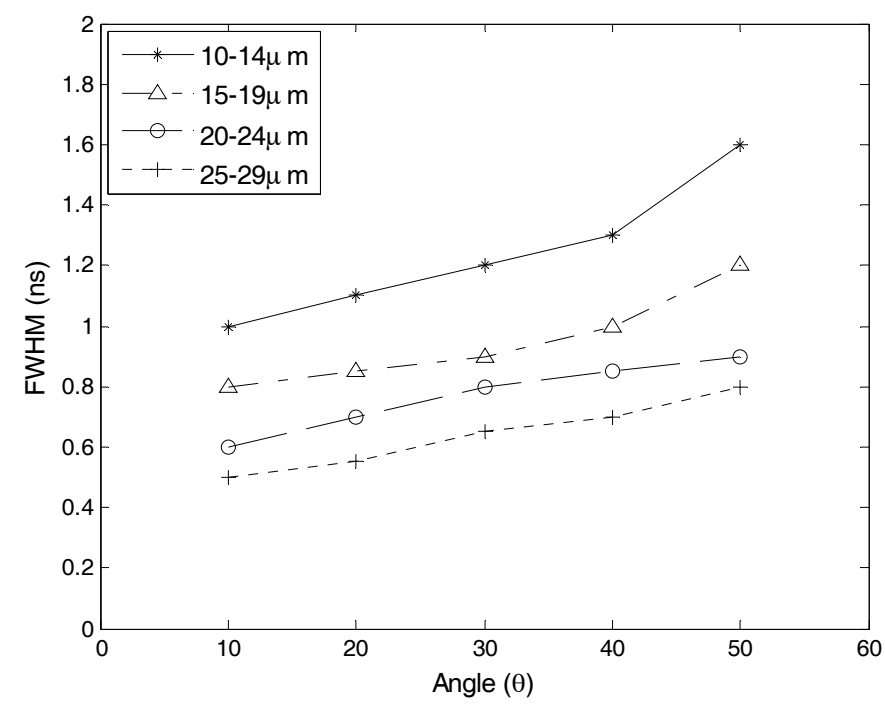

Figure 5. FWHM of the response of the $61.5 / 125 \mu \mathrm{m}$ fiber with different angles of launch $\theta$ and offsets

Figure 5 shows the resulting FWHM of the response of the fiber with respect to $\theta$ angle considering offsets at $10-14 \mu \mathrm{m}, 15-19 \mu \mathrm{m}, 20-24 \mu \mathrm{m}$, and $25-29 \mu \mathrm{m}$. It is also clear from Figure 5 that for the same offset as the coupling angle increases the propagation delay difference increases because the helix pitch increases which in turn make the traveled distance difference more. Bandwidth of the fiber can be approximated from this result by assuming Gaussian response of the fiber shown in Figure 4, which is a good approximation. If the fiber response is given by Equation (3), the resulting bandwidth will be the one given in Equation (4). To relate that to the FWHM of the response we use Equation (5).

$$
h(t)=\frac{1}{\sigma \sqrt{2 \pi}} e^{-\frac{t^{2}}{2 \sigma}}
$$




$$
\begin{aligned}
& B W=\frac{0.1323}{\sigma} \\
& B W=\frac{0.3115}{F W H M}
\end{aligned}
$$

Using Equation (5) the resulting BW of the fiber by conditioning the launch to be at angle $\theta=10$ degrees and an offset of 25-29 $\mu \mathrm{m}$ from the center of the fiber core is about $660 \mathrm{MHz} . \mathrm{km}$. Comparing this results with the fiber BW when used with VCSEL with no launch conditioning, the presented launch scheme gives four times better bandwidth. By comparing the presented launch scheme with previous work that has been reported in the literature such as Raddatz (1998), Raddatz (1999) and Webster (1999), it almost has the same bandwidth improvement, but with no additional expensive components such as single mode fiber patch cord to condition the launch.

\section{Conclusion}

This paper reported a launch scheme to be used for launching light from an $850 \mathrm{~nm}$ laser that produces a $4 \mu \mathrm{m}$ beam waist into $62.5 / 125 \mu \mathrm{m}$ GI fiber. The scheme involves offset from the center of the fiber core and an angle with the transverse plane of the fiber that makes the projection of the ray into the face of the fiber perpendicular to the radial vector. The presented launch scheme has been shown to ensure excitation of only circular helical modes of the fiber. An improvement in the capacity of GI fibers when selectively excite few number of helical modes of propagation was demonstrated.

\section{Acknowledgements}

S Al-Sowayan would like to thank Al Imam Mohammad Ibn Saud Islamic University for supporting this work, also would like to acknowledge Mr. Mohammed Asad Ali from Electrical Engineering Department at Al Imam Mohammad Ibn Saud Islamic University for helping in organizing the article.

\section{References}

Amphawan, A., Payne, F., O’Brien, D., \& Shah, N. (2010). Derivation of an Analytical Expression for the Power Coupling Coefficient for Offset Launch Into Multimode Fiber. J. of Lightwave Tech, 28(6), 861-869. http://dx.doi.org/10.1109/JLT.2009.2034475

Coleman, D. (2006). Migration to 50/125- 4 m in Local Area Network. Hicory, NC, Mar. 2006, Corning Cable Systems.

Gu, X. J., Mohammed, W., \& Smith, P. W. (2006). Demonstration of all-fiber WDM for multimode fiber local area networks. IEEE Photon. Technol. Lett., 18(1), 244-246. http://dx.doi.org/10.1109/LPT.2005.861538

Hahn, K. H. (1995). POLO-parallel optical links for Gigabit/s data communications. Proc. LEOS|'95 (pp. 228-229).

McInally, V. (2002). Multimode Fibre: The Progression towards Multi-Gigabit Speeds \& The 850 nm Operating Wavelength. Corning U.K.

Murshid, S., Grossman, B., \& Narakorn, P. (2008). Spatial domain multiplexing: A new dimension in fiber multiplexing. Optics and Laser Technology, 40(8), 995-1104. http://dx.doi.org/10.1016/j.optlastec.2008.03.001

Raddatz, L., White, I. H., Cunningham, D. G., \& Nowell, M. C. (1998). An experimental and theoretical study of the offset launch technique for the enhancement of the bandwidth of multimode fiber links. J. of Lightwave Technol., 16, 324-331. http://dx.doi.org/10.1109/50.661357

Raddatz, L., \& White, I. H. (1999). Overcoming the modal bandwidth limitation of multimode fiber by using passband modulation. IEEE Photonics Technol. Lett., 11(2), 266-268. http://dx.doi.org/10.1109/68.740725

Sargent, L. J., Webster, M., White, I. H., Heard, P. J., Penty, R. V., Tan, M. R. T., \& Cunningham, D. G. (1998). Simple technique for bandwidth enhancement of multimode fiber links using controlled spatial emission from vertical cavity surface emitting lasers. Electron. Lett., 34(21), 2038-2040. http://dx.doi.org/10.1049/el:19981417

Tsekrekos, C. P., Koonen, A. M. J., \& van den Boom, H. P. A. (2004). Selective Excitation of Graded Multimode Fibers Resulting in Annular Near Field Patterns. Proceedings Symposium IEEE/LEOS Benelux Chapter, 2004, Ghent. 
Webster, M., Raddatz, L., White, I. H., \& Cunningham, D. G. (1999). A statistical analysis of conditioned launch for gigabit ethernet links using multimode fiber. J. of Lightwave Tech, 17(9), 1532. http://dx.doi.org/10.1109/50.788558

\section{Copyrights}

Copyright for this article is retained by the author(s), with first publication rights granted to the journal.

This is an open-access article distributed under the terms and conditions of the Creative Commons Attribution license (http://creativecommons.org/licenses/by/3.0/). 